Pacific Journal of Mathematic 


\title{
ON THE EXISTENCE OF SUPPORT POINTS OF SOLID CONVEX SETS
}

\author{
J. T. MARTI
}

Let $E$ be a separable Frechet lattice. It is shown that a solid convex set $X$ with void interior in $E$ is supported at each of its boundary points if and only if the span of $X$ is not dense in $E$. This result then is applied to the case of solid convex sets with void interior in real Fréchet spaces with an unconditional Schauder basis and in the real Banach lattice $C(S)$, $S$ compact Hausdorff.

1. Introduction. If $E$ is a real Hausdorff topological vector space and $X$ is a convex subset of $E$ with nonempty interior and boundary $\partial X$ then, by a known theorem, every point of $\partial X$ supports $X$, that is, for every $x \in \partial X$ there exists a continuous nontrivial linear functional $f$ on $E$ such that $\sup f(X)=f(x)$. However, if $X$ has void interior, there are examples of compact convex sets, e.g., the Hilbert cube in $l_{2}[1, \mathrm{p} .160]$, which have boundary points that are not support points.

The object of this note is to investigate conditions on convex sets $X$ with void interior in a separable real Fréchet lattice $E$, such that every point of $\partial X$ is a support point of $X$. A theorem obtained is that for such sets $X$ which are also solid, $X$ is supported at each boundary point if and only if the span sp $X$ of $X$ is not dense in $E$. Moreover, if $E$ is a real Fréchet space with an unconditional basis $\left\{x_{n}, f_{n}\right\}$ (the sequence space $s$, the Banach spaces $c_{0}$ and $l_{p}(1 \leqq p<\infty$ ) and so all separable real Hilbert spaces are examples of such spaces) and if $E$ is equipped with the ordering induced by the basis $\left\{x_{n}, f_{n}\right\}$, then a solid convex set $X$ with void interior in $E$ is supported at each of its boundary points if and only if $\bar{X}$ does not contain a weak order unit of $E$. On the other hand, if $E$ is the Banach lattice $C(S), S$ compact Hausdorff, all solid convex subsets $X$ with void interior in $E$ have the property that the boundary points and the support points of $X$ coincide.

2. Support properties of solid convex sets with void interior. A set $X$ in a Fréchet lattice $E$ is said to be solid if $y$ is in $X$ whenever $x$ is in $X$ and $|y| \leqq|x|$. An element $x$ in the positive cone of $E$ is said to be a weak order unit of $E$ if $y=0$ whenever $y$ is in $E$ and $x \wedge|y|=0$. For the terminology see also H. H. Schaefer [5] or A. L. Peressini [4]. The topological boundary of $X$ is denoted by $\partial X$. 
THEOREM 1. Let $X$ be a solid convex set with void interior in a separable real Fréchet lattice $E$. Then every $x \in \partial X$ supports $X$ if and only if $\mathrm{sp} X$ is not dense in $E$.

Proof. (Sufficiency) Let $E^{\prime}$ be the topological dual of $E$. If $\operatorname{sp} X$ is not dense in $E$ there is an $f \in E^{\prime} \backslash\{0\}$ such that $f(X)=\{0\}$. In this case $f$ obviously is a supporting functional of $X$ for every $x \in \partial X$.

(Necessity) Let $K$ be the positive cone of $E$ and $K^{\prime}=\left\{f \in E^{\prime}: f(x) \geqq\right.$ $0, x \in K\}$ the dual cone in $E^{\prime}$. We define the sets $S_{x}, x \in X \cap K$, by

$$
S_{x}=\left\{f \in K^{\prime}: f(x)=0\right\} .
$$

It is clear that each $S_{x}$ is a $\sigma\left(E^{\prime}, E\right)$-closed set in $E^{\prime}$ which contains 0. Moreover, let

$$
S=\bigcap_{x \in X \cap K} S_{x} .
$$

Since $E$ is a Fréchet space there exists a countable base $\left\{U_{n}\right\}$ of neighborhoods of 0 in $E$, and since $E$ is separable there is a sequence $\left\{V_{n}\right\}$ of open $\sigma\left(E^{\prime}, E\right)$-neighborhoods of 0 in $E^{\prime}$ satisfying $\bigcap_{n=1}^{\infty} V_{n}=\{0\}$. (The sequence $\left\{V_{n}\right\}$ can, for instance, be constructed in the following way: If $\left\{x_{n}\right\}$ is a dense set in $E$, let $W_{m n}$ be defined by $W_{m n}=$ $\left\{f \in E^{\prime}:\left|f\left(x_{n}\right)\right|<1 / m\right\}$. It then follows that $\bigcap_{m, n=1}^{\infty} W_{m n}=\{0\}$ since for each $f$ in this last intersection one has $f\left(\left\{x_{n}\right\}\right)=\{0\}$ and hence $f=0$.) We assume now that $S=\{0\}$. Then $E^{\prime} \backslash\{0\}=\complement S$ and so for all $m, n \in N$ one has

$$
U_{n}^{0} \subset \bigcup_{k=1}^{\infty} U_{k}^{0}=E^{\prime}=V_{m} \cup \bigcup_{x \in X \cap K}\left\lceil S_{x} .\right.
$$

Since the polars $U_{n}^{0}$ of $U_{n}$ are $\sigma\left(E^{\prime}, E\right)$-compact there is for each $m$ and each $n$ in $N$ a finite set $A_{m n}$ in $X \cap K$ such that

$$
U_{n}^{0} \subset V_{m} \cup \bigcup_{x \in A_{m n}}\left\lceil S_{x} .\right.
$$

If $\left\{x_{k}\right\}$ is a sequence in $X \cap K$ such that $\left\{x_{k}\right\}=\bigcup_{m, n=1}^{\infty} A_{m n}$ we get for all $m \in N$,

$$
E^{\prime}=\bigcup_{n=1}^{\infty} U_{n}^{0}=V_{m} \cup \bigcup_{k=1}^{\infty}\left\lceil S_{x_{k}} .\right.
$$

Whence

$$
E^{\prime}=\bigcap_{m=1}^{\infty}\left(V_{m} \cup \bigcup_{k=1}^{\infty}\left\lceil S_{x_{k}}\right)=\bigcup_{k=1}^{\infty}\left\lceil S_{x_{k}} \cup\{0\}\right.\right.
$$

and

$$
\bigcap_{k=1}^{\infty} S_{x_{k}}=\complement\left(\bigcup_{k=1}^{\infty} \complement S_{x_{k}}\right)=\complement\left(E^{\prime} \backslash\{0\}\right)=\{0\} .
$$


Next, if $d$ is a translation invariant metric generating the topology of $E$, we define the real sequence $\left\{a_{k}\right\}$ by

$$
a_{k}=\inf \left\{2^{-k}, \sup \left\{t>0: d\left(0, s x_{k}\right) \leqq 2^{-k}, s \in[0, t]\right\}\right\} .
$$

Since $X$ is solid we have $\left(2^{n}-1\right) 2^{-n+k} a_{k} x_{k} \in X$ for all $k, n \in N$ and since $X$ is convex,

$$
\sum_{k=1}^{n} a_{k} x_{k}=\left(2^{n}-1\right)^{-1} 2^{n} \sum_{k=1}^{n} 2^{-k}\left(2^{n}-1\right) 2^{-n+k} a_{k} x_{k} \in X
$$

for all $n \in N$. Since $\bar{X}$ is complete and since for $n<m$

$$
d\left(\sum_{k=1}^{n} a_{k} x_{k}, \sum_{k=1}^{m} a_{k} x_{k}\right) \leqq \sum_{k=n+1}^{m} d\left(0, a_{k} x_{k}\right) \leqq \sum_{k=n+1}^{m} 2^{-k}<n^{-1},
$$

$\lim _{n} \sum_{k=1}^{n} a_{k} x_{k}$ exists in $\bar{X}$ and this limit is denoted by $x$. Since int $X=\varnothing$ and $\bar{X}$ is solid [4, Proposition 2.4.8] it follows that $1 / 2 x \in \partial X$ and thus is a support point of $X$. If $f$ is a corresponding support functional we have $f \neq 0$ and $0=f(0) \leqq f(1 / 2 x)=f(x)-$ $f(1 / 2 x)$. If $\left\{y_{n}\right\} \subset X$ is a sequence that converges to $x$ in $E$ one obtains $f(x) \leqq \sup _{n} f\left(y_{n}\right) \leqq f(1 / 2 x)$, and hence $f(x)=0$. Now, since $E$ is a locally convex lattice and again since $\bar{X}$ is solid, it follows that

$$
\begin{aligned}
0 & \leqq|f|(x) \\
& \leqq \sup \{f(y): y \in E,|y| \leqq x\} \leqq \sup f(\bar{X})=\sup f(X)=f\left(\frac{1}{2} x\right)=0 .
\end{aligned}
$$

Therefore, $|f| \in K^{\prime} \backslash\{0\}$ and $|f|(x)=0$. This shows that

$$
S_{x} \backslash\{0\} \neq \varnothing \text {. }
$$

Since $0 \leqq a_{k} x_{k} \leqq x$, one has $0 \leqq g\left(x_{k}\right) \leqq a_{k}^{-1} g(x)=0, g \in S_{x}, k \in N$. In view of (1) one thus obtains

$$
\{0\} \subset S_{x} \subset \bigcap_{k=1}^{\infty} S_{x_{k}}=\{0\},
$$

and this contradiction shows that

$$
S \backslash\{0\} \neq \varnothing \text {. }
$$

If $f$ is a nonzero element of $S$ then $f(X \cap K)=\{0\}$. Thus for any $x \in X$ we have $f(x)=f\left(x^{+}\right)-f\left(x^{-}\right)=0$ because $x^{ \pm} \in X$. Hence $f(X)=$ $\{0\}$, showing that sp $X$ cannot be dense in $E$.

Let now $E$ be a real Fréchet space with an unconditional basis $\left\{x_{n}, f_{n}\right\}$. It is known that the set $K=\left\{x \in E: f_{n}(x) \geqq 0, n \in N\right\}$ is a closed, normal, generating cone in $E$ and equipped with $K, E$ becomes an order complete locally convex lattice [3, Theorem 5]. Obviously, $\left\{x_{n}\right\} \subset K$ and the coefficient functionals $f_{n}$ are positive with respect to $K$. Therefore, the basis is a positive Schauder basis for $E$ [2]. 
REMARK. A slight modification of the above argument shows that in Theorem 1 the separability of $E$ can be replaced by the (weaker) condition: There exists a sequence $\left\{u_{n}\right\}$ in the positive cone of $E$ such that sp $\bigcup_{n=1}^{\infty}\left[0, u_{n}\right]$ is dense in $E$.

THEOREM 2. If $X$ is a solid convex set with void interior in $E$ ( $E$ being specified above), then every point of $\partial X$ supports $X$ if and only if $\bar{X}$ does not contain a weak order unit of $E$.

Proof. (Necessity) Let every point of $\partial X$ support $X$ and let us assume that $\bar{X}$ contains a weak order unit $x$ of $E$. Then from [3, Proposition 11] it follows that the span of $[0, x]$ is dense in $E$. Since $[0, x] \subset \bar{X}$ this contradicts Theorem 1 . Hence $\bar{X}$ does not contain a weak order unit.

(Sufficiency) If $\bar{X}$ does not contain a weak order unit of $E$, suppose that $\sup f_{n}(X)>0, n \in N$. Then for every $n$ there is a $y_{n} \in X$ such that $\sup f_{n}(X) \leqq 2 f_{n}\left(y_{n}\right)$. Since $X$ is solid this yields for all $n$ that $\sup f_{n}(X) x_{n} \leqq 2\left|y_{n}\right|$; whence $1 / 2 \sup f_{n}(X) x_{n} \in X$. In the same way as in the proof of the necessity part of the preceding theorem we can now construct an element $x \in \bar{X} \cap K$ such that $x=\lim _{n} \sum_{i=1}^{n} a_{i} x_{i}$, where $a_{i}>0, i \in N$. If $y \in K \backslash\{0\}$ then there must be a positive integer $n$ such that $f_{n}(y)>0$. If $z \in K$ is given by $z=\inf \left\{a_{n}, f_{n}(y)\right\} x_{n}$ it follows that $z \neq 0$ and $z=x \wedge y$, i.e., $x$ is a weak order unit of $E$ in $\bar{X}$. By this contradiction to our assumption there is an $n \in N$ such that $\sup f_{n}(X)=0$. Therefore, $\operatorname{sp} X$ cannot be dense in $E$ and an application of Theorem 1 finally completes the proof.

Concerning the real Banach lattice $C(S), S$ compact Hausdorff, it is clear that there can exist solid subsets $X$ of $C(S)$ with void interior containing a weak order unit of $C(S)$ and such that every boundary point of $X$ is a support point of $X$. For instance, take $X=\{y \in C[0,1]:|y| \leqq x\}$, where $x$, given by $x(s)=s, s \in[0,1]$, is a weak order unit of $C[0,1]$. Therefore, that $\bar{X}$ contains no weak order unit of $C(S)$ is not a necessary condition for $X$ to be supported at each boundary point, as is also seen by the following theorem:

THEOREM 3. If $X$ is a convex solid set with void interior in $C(S)$ then every boundary point of $X$ supports $X$.

Proof. We assume that $\mathrm{sp} X$ is dense in $C(S)$. If $f_{\mathrm{s}}$ is the point evaluation functional of a general point $s$ of $S$, this implies that $\sup f_{s}(X)>0, s \in S$. In this case, since $X$ is solid, there is for every $s \in S$ an $x_{s} \geqq 0$ in $X$ such that $x_{s}(s)>0$. Hence for every $s \in S$ 
there is an open neighborhood $V_{s}$ of $s$ in $S$ such that inf $x_{s}\left(V_{s}\right)>0$. Since $S$ is compact and $\left\{V_{s}: s \in S\right\}$ is an open covering of $S$ there is a finite subcovering $\left\{V_{s(1)}, \cdots, V_{s(m)}\right\}$ for $S$. Taking $x=m^{-1} \sum_{n=1}^{m} x_{s(n)}$ it is clear that $x$ is in $X$ since $X$ is convex, and that

$$
\inf x(S) \geqq m^{-1} \inf _{n \leqq m} \inf x_{s(n)}\left(V_{s(n)}\right)>0 .
$$

If $U$ is the unit ball of $C(S)$ we obtain (inf $x(S))|y| \leqq x, y \in U$, which, since $X$ is solid, implies that $(\inf x(S)) U \subset X$. This contradiction shows that $\overline{\mathrm{sp}} X \neq C(S)$ and the result follows in the same way as in the sufficiency part of the proof of Theorem 1 .

\section{REFERENCES}

1. N. Bourbaki, Espaces vectorielles topologiques III-V, Hermann et Cie., 1955.

2. J. T. Marti, On positive bases in ordered topological vector spaces, Arch. d. Math., 22 (1971), 657-659.

3. _- On locally convex spaces ordered by a basis, Math. Ann., 195 (1971), 79-86. 4. A. L. Peressini, Ordered Topological Vector Spaces, Harper and Row, New York, 1967.

5. H. H. Schaefer, Topological Vector Spaces, Macmillan, New York, 1966.

Received November 8, 1972 and in revised form August 10, 1973.

Current Address: J. T. Marti

Eidg. Technische Hochschule

Seminar für Angewandte Mathematik

Clausiusstrasse 55

CH-8006 Zürich, Switzerland 



\section{PACIFIC JOURNAL OF MATHEMATICS}

\section{EDITORS}

RICHARD ARENS (Managing Editor)

University of California

Los Angeles, California 90024

R. A. BeAumont

University of Washington

Seattle, Washington 98105
J. DugundJI*

Department of Mathematics

University of Southern California

Los Angeles, California 90007

D. Gilbarg and J. Milgram

Stanford University

Stanford, California 94305

\section{ASSOCIATE EDITORS}

E. F. BECKENBACH

B. H. NEUMANN

F. WOLF

K. YoSHIDA

\section{SUPPORTING INSTITUTIONS}

UNIVERSITY OF BRITISH COLUMBIA
CALIFORNIA INSTITUTE OF TECHNOLOGY
UNIVERSITY OF CALIFORNIA
MONTANA STATE UNIVERSITY
UNIVERSITY OF NEVADA
NEW MEXICO STATE UNIVERSITY
OREGON STATE UNIVERSITY
UNIVERSITY OF OREGON
OSAKA UNIVERSITY

UNIVERSITY OF BRITISH COLUMBIA CALIFORNIA INSTITUTE OF TECHNOLOGY UNIVERSITY OF CALIFORNIA MONTANA STATE UNIVERSITY NEW MEXICO STATE UNIVERSITY UNIVERSITY OF OREGON OSAKA UNIVERSITY
UNIVERSITY OF SOUTHERN CALIFORNIA STANFORD UNIVERSITY UNIVERSITY OF TOKYO UNIVERSITY OF UTAH WASHINGTON STATE UNIVERSITY UNIVERSITY OF WASHINGTON AMERICAN MATHEMATICAL SOCIETY NAVAL WEAPONS CENTER

* C. R. DePrima California Institute of Technology, Pasadena, CA 91109, will replace J. Dugundji until August 1974. 


\section{Pacific Journal of Mathematics}

\section{Vol. 51, No. $1 \quad$ November, 1974}

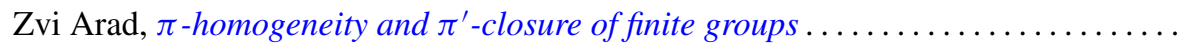

Ivan Baggs, A connected Hausdorff space which is not contained in a maximal

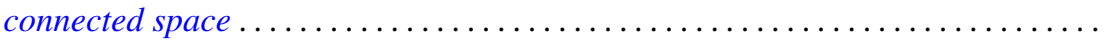

Eric Bedford, The Dirichlet problem for some overdetermined systems on the unit ball in $C^{n}$

R. H. Bing, Woodrow Wilson Bledsoe and R. Daniel Mauldin, Sets generated by

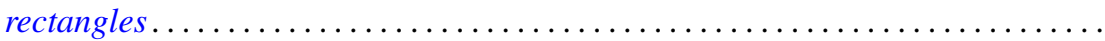

Carlo Cecchini and Alessandro Figà-Talamanca, Projections of uniqueness for

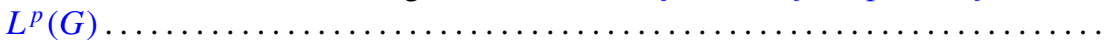

Gokulananda Das and Ram N. Mohapatra, The non absolute Nörlund summability of Fourier series .

Frank Rimi DeMeyer, On separable polynomials over a commutative ring ........ Richard Detmer, Sets which are tame in arcs in $E^{3} \ldots \ldots \ldots \ldots \ldots \ldots \ldots \ldots$

William Erb Dietrich, Ideals in convolution algebras on Abelian groups ..........

Bryce L. Elkins, A Galois theory for linear topological rings .................

William Alan Feldman, A characterization of the topology of compact convergence on $C(X)$.

Hillel Halkin Gershenson, A problem in compact Lie groups and framed cobordism

Samuel R. Gordon, Associators in simple algebras.

Marvin J. Greenberg, Strictly local solutions of Diophantine equations

Jon Craig Helton, Product integrals and inverses in normed rings . . . . . . . . . . . .

Domingo Antonio Herrero, Inner functions under uniform topology . . .

Jerry Alan Johnson, Lipschitz spaces .

Marvin Stanford Keener, Oscillatory solutions and multi-point boundary value

functions for certain nth-order linear ordinary differential equations.

John Cronan Kieffer, A simple proof of the Moy-Perez generalization of the

Shannon-McMillan theorem .......................

Joong Ho Kim, Power invariant rings

Gangaram S. Ladde and V. Lakshmikantham, On flow-invariant sets .

Roger T. Lewis, Oscillation and nonoscillation criteria for some self-adjoint even

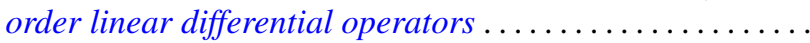

Jürg Thomas Marti, On the existence of support points of solid convex sets ..

John Rowlay Martin, Determining knot types from diagrams of knots . .

James Jerome Metzger, Local ideals in a topological algebra of entire functions

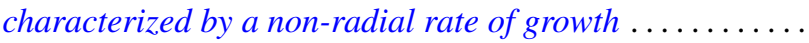

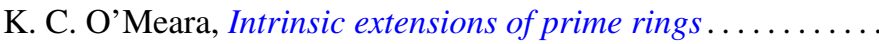

Stanley Poreda, A note on the continuity of best polynomial approximations ..

Robert John Sacker, Asymptotic approach to periodic orbits and local prolongations

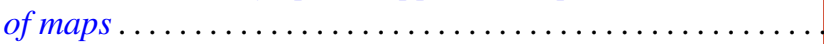

Eric Peter Smith, The Garabedian function of an arbitrary compact set . .

Arne Stray, Pointwise bounded approximation by functions satisfying a side condition

John St. Clair Werth, Jr., Maximal pure subgroups of torsion complete abelian

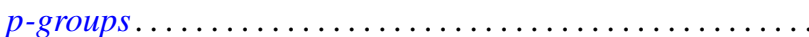

